include student training in dementia basics and communication strategies, regularly scheduled social contacts and journaling about the experience. Data analysis includes thematic examination of journal data and pre-post measures of satisfaction, knowledge, attitudes and caregiver burden. Results: The Northwestern program has recruited 241 dyads. The MAC has enrolled 33 students across disciplines. At Columbia, the program has trained and paired 100 students. The University of Minnesota/Health Partners program has enrolled 37 dyads. Journal submissions demonstrate changing beliefs and increased understanding of dementia and its impact on the person and family. Pre- and posttest data reveal increases in knowledge of dementia, empathy, positive attitudes and decreased stigma among medical students. Evaluations from PWD and their families reveal an opportunity to highlight remaining strengths and remain socially engaged while contributing to a young person's education. Conclusions: The original replicable experiential program, previously validated targeting medical students, has been adapted in multiple settings to meet the needs of undergraduate and graduate students with similar positive results for the student, person with dementia and family.

\section{O4-03-05 INTERPROFESSIONAL TEAM TRAINING FOR EARLY IDENTIFICATION OF ADRD}

Jennifer Lingler ${ }^{1}$, Elizabeth Mulvaney ${ }^{2}$, Clayton Jacobs ${ }^{3}$, Saskia Berrio-Thomas ${ }^{4}$, Jonna M. Morris ${ }^{5},{ }^{1}$ University of Pittsburgh, Pittsburgh, PA, USA; ${ }^{2}$ University of Pittsburgh School of Social Work, Pittsburgh, PA, USA; ${ }^{3}$ Alzheimer's Association Greater PA Chapter, Pittsburgh, PA, USA; ${ }^{4}$ University of Michigan, Ann Arbor, MI, USA, ${ }^{5}$ University of Pittsburgh School of Nursing, Pittsburgh, PA, USA. Contact e-mail: linglerj@pitt.edu

Background: Mounting empirical evidence supports making interprofessional team-based care the standard of practice for dementia. Few health professional training programs offer interprofessional clinical rotations to prepare trainees for roles in collaborative care practice. Our objective is to describe the implementation and evaluation of an interprofessional team training experience focused on the early identification and detection of Alzheimer's disease and related disorders (ADRD). Methods: Trainees from social work ( $S W ; n=6)$, nurse practitioner $(\mathrm{NP} ; \mathrm{n}=2)$, and psychiatry residency $(\mathrm{MD} ; \mathrm{n}=10)$ programs completed an average of 96 hours of clinical training in teambased approaches to the assessment of ADRD at the University of Pittsburgh Alzheimer Disease Research Center. Pre and post rotation surveys evaluated trainees' attitudes toward interprofessional teamwork and geriatrics, and perceived skillfulness in assessment and intervention for early stage ADRD. A subset of trainees $(n=4)$ participated in a focus group designed to provide in-depth feedback. Results: Analyses of surveys showed little change in attitudes toward teamwork and geriatrics, both of which were high at baseline. Perceived skillfulness in ADRD care increased among NP and MD trainees, with statistically significant gains for 13/15 items measured including: "evaluating signs and symptoms of dementia noting pertinent positives and negatives" ( $\mathrm{t}=-6.71 ; \mathrm{df}=5 ; \mathrm{p}=.001)$; "differentiating between normal and abnormal changes associated with aging" ( $\mathrm{t}=-3.16$; $\mathrm{df}=5 ; \mathrm{p}=.025)$; and "recognizing the presence of co-morbidities, their impact on presenting health problems, and the risk for iatrogenesis" $(\mathrm{t}=-5.00 ; \mathrm{df}=5 ; \mathrm{p}=.004)$. Perceived skillfulness also increased among SW trainees with significant gains in 10/12 items, including: "assisting caregivers to reduce stress levels and maintain mental and physical health" $(\mathrm{t}=-3.87 ; \mathrm{df}=5$; $\mathrm{p}=.012)$ and "providing anticipatory guidance and counseling to individuals and families" $(\mathrm{t}=-2.71 ; \mathrm{df}=5 ; \mathrm{p}=.042)$. Focus group analysis reinforced findings of positive interprofessional and clinical skill development which trainees attributed to the logistics of the setting, including mentorship. Trainees voiced confidence about implementing newly acquired clinical skills in future care settings, but were less clear on how to transfer interprofessional team collaboration to other settings. Conclusions: Interprofessional training may promote skillfulness in core competencies for ADRD care. Additional exposure to exemplar interprofessional teams may increase confidence that teamwork skills transfer to practice.

\begin{tabular}{|l|l}
\hline O4-03-06 & A RANDOMIZED CONTROLLED TRIAL \\
EVALUATING THE IMPACT OF AN \\
E-LEARNING INTERVENTION BASED ON \\
THE IMPROVING WELL-BEING AND \\
HEALTH FOR PEOPLE WITH DEMENTIA \\
(WHELD) PERSON-CENTRED CARE \\
TRAINING PROGRAMME
\end{tabular}

Joanne McDermid ${ }^{1}$, Jane Fossey ${ }^{2}$, Miguel Vasconcelos Da Silva ${ }^{1}$, Clive Ballard ${ }^{3},{ }^{1}$ King's College London, London, United Kingdom; ${ }^{2}$ Oxford Health NHS Foundation Trust, Oxford, United Kingdom; ${ }^{3}$ University of Exeter Medical School, Exeter, United Kingdom. Contact e-mail: miguel.1. dasilva@kcl.ac.uk

Background: Affordable, high-quality and evidence-based dementia training for nursing home staff is a high priority. We report a randomized controlled trial (RCT) of an e-learning programme based on our improving Wellbeing and Health for People with Dementia (WHELD) person-centred care training. Methods: A randomised single blind clinical trial comparing the online WHELD intervention with and without Research Therapist support, as well as enhanced treatment as usual. It took place in 24 London care homes with over 250 care staff. All care homes taking part in the online training programme received 5 interactive, multimedia online modules developed specifically for care home staff working with people with dementia (PwD). They covered key WHELD programme elements of person-centered care, social interaction and antipsychotic review. One third of care homes received the online training plus light touch support from a Research Therapist, one third received online training alone and the remaining third received enhanced treatment as usual. Results: Primary outcome measure: staff attitudes towards people with dementia (Approaches to Dementia Questionnaire), a standardised and well validated questionnaire. Other outcomes: quality of care and interactions between staff and residents, staff work stress and satisfaction in caring for PwD and staff knowledge of dementia. This is the largest RCT of an online person-centred care training programme for staff working in care homes with people with dementia. Preliminary analysis of the primary outcome indicated a significant positive benefit of staff attitudes related to person centred care $(t=2.29$ $\mathrm{p}=0.004$ ), demonstrating the value and feasibility of this approach. No benefits were seen in the group who did not receive additional training or in the group who received the online intervention alone. Conclusions: The results are encouraging and suggest the WHELD intervention delivered as an e-learning programme with skype supervision delivers significant benefits for care staff. Further work is needed to evaluate the benefits for residents with dementia. 